\title{
A tribute to Mats Gyllenberg, on the occasion of his 60th birthday
}

\author{
Jukka Corander ${ }^{1}$. Odo Diekmann² . \\ Timo Koski ${ }^{3}$
}

Published online: 27 January 2016

(C) Springer-Verlag Berlin Heidelberg 2016

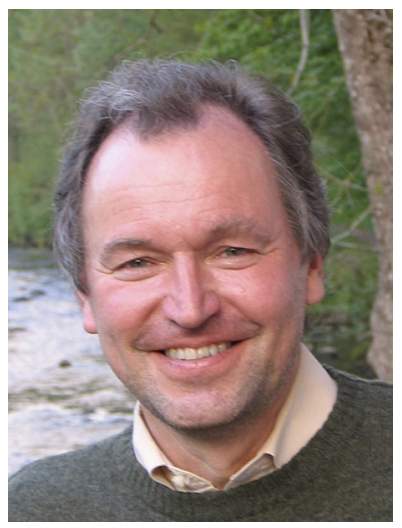

The footprints of a scientist are found in journals, books and folklore and are amplified by students and others that follow the master's footsteps. In Mathematical Biology, Mats Gyllenberg's footprints abound. His sixtieth birthday triggers us to show our appreciation of his manifold achievements and to express our gratitude for the many ways in which he has inspired people and stimulated developments.

In 1987 Mats received the degree of Doctor of Technology at Helsinki University of Technology (with the highest grade: 'laudatur'), but already in 1981 he published a paper in the Proceedings of the American Mathematical Society on Volterra integral equations, while in 1982 his first paper with 'population dynamics' in the title appeared in Mathematical Biosciences. These two themes, infinite dimensional dynamical systems and population dynamics, are recurrent. The link between them is 'structure', age structure at first, but soon other forms of structure received attention too. Mats'

\footnotetext{
$凶$ Odo Diekmann

o.diekmann@uu.nl

Jukka Corander

jukka.corander@helsinki.fi

Timo Koski

tjtkoski@kth.se

1 Department of Mathematics and Statistics, University of Helsinki, Helsinki, Finland

2 Mathematical Institute, Utrecht University, Utrecht, The Netherlands

3 Department of Mathematics, KTH Royal Institute of Technology, Stockholm, Sweden
} 


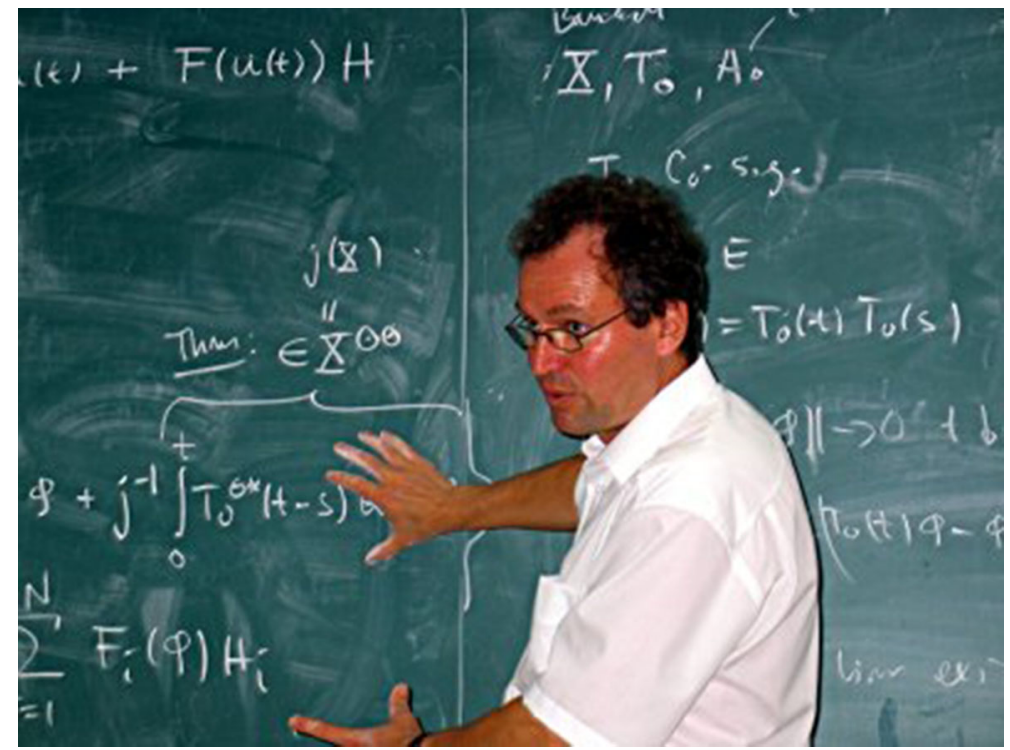

"Mats Gyllenberg in action, explaining sun-star calculus"

first paper in the Journal of Mathematical Biology exemplifies this, it dates from 1986 and deals with the size and scar distribution of the yeast Saccharomyces cerevisiae.

Mats and I (= Odo Diekmann) met in early July 1983 when Mats came for a short visit to the Mathematisch Centrum (later rebaptized 'Centre for Mathematics and Computer Science') in Amsterdam. That same month we met again, at an influential meeting in Bari, organized by Vincenzo Capasso and others. Upon hearing that Mats was thinking about spending a year abroad, I proposed that he come to Amsterdam. Both Mats and Horst Thieme spent the academic year 84-85 at the Mathematisch Centrum, to join forces with Hans Metz, Henk Heijmans and myself in developing models of physiologically structured populations. Much effort was put into the infinite dimensional dynamical systems perspective. Joint work with Philippe Clément (Delft University of Technology) culminated in sun-star calculus for adjoint semigroups.

The next academic year (85-86), Mats visited Glenn Webb at Vanderbilt University and worked with him on cell population models involving quiescence. Then Mats returned to Helsinki and stayed there until in 1989 he was appointed Professor of Applied Mathematics at the University of Luleå in Sweden, where he met Timo Koski, marking the start of a long time cooperation on numerical taxonomy and its mathematical ramifications. In 1992 he moved to the University of Turku and in 2004 to the University of Helsinki, where he presently is Head of the Department of Mathematics.

It is impossible to list all the executive functions that Mats had and has, the sheer number is forbidding. Where others would shy away from management tasks in national or international organizations, an appeal to Mats is seldom (never ?) in vain. The acronyms EMS, IIASA, ESF, ICIAM, ERC, ESMTB are scattered all over his 
$\mathrm{CV}$ and these do not cover the many Finnish and Nordic organizations that appear throughout. As a member or chairman of organizing or scientific committees of meetings too, Mats has done a tremendous service to the community. And of course we should also mention that, after becoming a member of the editorial board of the Journal of Mathematical Biology in 2000, Mats accepted to take on the demanding role of managing editor in 2009.

Mats is not only a prolific writer, as testified by the multitude of his scientific papers, his wide-ranging interests and detailed knowledge of a broad array of subjects allow him to write in an attractive and entertaining manner. [To anyone who wonders what we mean by this, we recommend to read the review of Horst Thieme's Mathematics in Population Biology, Math. Biosc. (2005) 193: 13-18.] Mats has written about many different topics. Particularly noteworthy are his joint papers with his father, the microbiologist Helge G. Gyllenberg, on numerical taxonomy of bacteria (enriching the time honoured subject with modern notions from statistical learning theory) and his work on metapopulations with Ilkka Hanski and later with Hans Metz. Other topics are snoring, evolutionary suicide, evolution of dispersal, parameter identification, fungal endophytes, limiting similarity, quasi-stationary distributions, limit cycles in polynomial systems, periodic solutions of competitive systems ...

To state that life begins at 60 is wishful thinking of someone even older. So let us wish Mats a second youth, a long period of less obligations, more time, more freedom and, most of all, much satisfaction from successful research. This is, in effect, a selfish wish because it will benefit the progress and the flourishing of Mathematical Biology. Meanwhile: thanks a lot Mats for all you have already done!

Helsinki, Utrecht, Stockholm, December 222015

Jukka Corander, Odo Diekmann, Timo Koski 